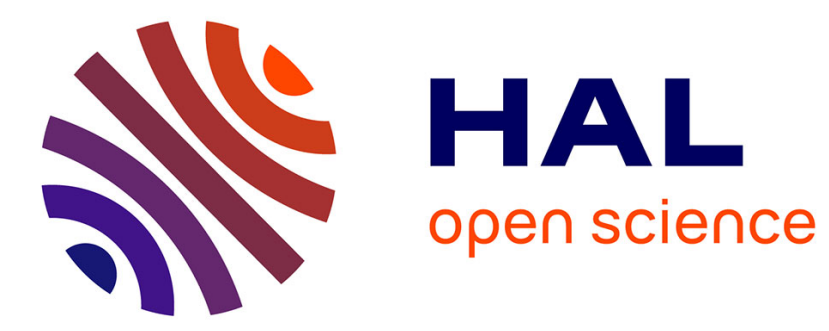

\title{
ÉLECTROVESTIBULOGRAPHIE PAR ÉLECTRODES À DISTANCE CHEZ L'ANIMAL
}

R. Charlet de Sauvage, B. Guerange, T. Lemettre, J. Aran, J. Erre

\section{To cite this version:}

R. Charlet de Sauvage, B. Guerange, T. Lemettre, J. Aran, J. Erre. ÉLECTROVESTIBULOGRAPHIE PAR ÉLECTRODES À DISTANCE CHEZ L'ANIMAL. Journal de Physique IV Proceedings, 1992, 02 (C1), pp.C1-177-C1-180. 10.1051/jp4:1992135 • jpa-00251206

\section{HAL Id: jpa-00251206 https://hal.science/jpa-00251206}

Submitted on 1 Jan 1992

HAL is a multi-disciplinary open access archive for the deposit and dissemination of scientific research documents, whether they are published or not. The documents may come from teaching and research institutions in France or abroad, or from public or private research centers.
L'archive ouverte pluridisciplinaire HAL, est destinée au dépôt et à la diffusion de documents scientifiques de niveau recherche, publiés ou non, émanant des établissements d'enseignement et de recherche français ou étrangers, des laboratoires publics ou privés. 


\title{
ÉLECTROVESTIBULOGRAPHIE PAR ELECTRODES À DISTANCE CHEZ L'ANIMAL
}

\author{
R. CHARLET de SAUVAGE, B. GUERANGE, T. LEMETTRE, J.M. ARAN et J.P. ERRE \\ Unité INSERM 229, Audiologie Expérimentale, Hôpital Pellegrin, Bât. P, F-33076 Bordeaux cedex, \\ France
}

\begin{abstract}
In order to produce synchronous detectable responses in the vestibular pathways, electrical stimuli (ES) were applied on guinea pigs cochleas, first during rest then during an angular acceleration. The difference between the signals recorded before and during rotation represented the variation of the response to ES induced by the kinetic excitation and thus represents an electro-vestibulographic (EVG) type of response. EVG responses recorded on the round window and on the vertex were made each of a main negative wave, with a rotation speed threshold of about 2 to $3 \% \mathrm{~s}$, with amplitudes proportional to the acceleration culminating respectively to $4 \mu \mathrm{V}$ and $40 \mu \mathrm{V}$ with latencies around $0.4 \mathrm{~ms}$ and $1.2 \mathrm{~ms}$, which could be associated with the vestibular nerve and nucleus. These results, along with those related to post rotation responses and to selective cochlear and vestibular destructions, confirmed the vestibular specificity of EVG responses.
\end{abstract}

\section{RESUME}

Afin de produire dans les voies vestibulaires des réponses synchrones observables, des stimulations électriques (SE) sont appliquées sur la cochlée du cobaye, au repos puis au cours d'une accélération angulaire. La différence entre les signaux enregistrés avant et pendant la rotation reflète la variation de la réponse à la SE induite par l'excitation cinétique et représente donc une réponse de type électro-vestibulographique (EVG). Les réponses EVG enregistrées sur la fenêtre ronde et le vertex comportent chacune une onde principale négative, de seuil en vitesse voisin de $2 \mathrm{à} 3 \%$, d'amplitude porportionnelle à l'accélération, et, respectivement, culminant à $40 \mu \mathrm{V}$ et $4 \mu \mathrm{V}$ avec des latences voisines de $0,4 \mathrm{~ms}$ et $1,2 \mathrm{~ms}$, qui peuvent être associées au nerf et au noyau vestibulaires. Ces résultats, ainsi que ceux relatifs aux réponses post rotatoires et à des destructions vestibulaires et cochléaires sélectives, confirment la spécificité vestibulaire des réponses EVG.

\section{INTRODUCTION}

A cause de la courbe de réponse de type basse fréquence du système vestibulaire, l'étalement temporel des décharges nerveuses ne permet de produire des potentiels vestibulaires discernables du bruit de fond que pour des accélérations situées très au-delà de la gamme physiologique (1). Dans les années récentes, une solution à cette difficulté a pu être trouvée. Elle consiste à stimuler simultanément toutes les fibres cochléo-vestibulaires à l'aide d'une stimulation électriques (SE) afin d'obtenir un potentiel évoqué observable. Pour extraire des composantes spécifiques, nous avons proposé une procédure consistant à enregistrer les réponses évoquées par la SE dans deux conditions d'excitabilité électrique du nerf produites par des stimulations sensorielles spécifiques. Nous avons déjà démontré l'efficacité de cette méthode pour le système auditif (2) et, récemment, pour le système vestibulaire en utilisant une électrode d'enregistrement sur le nerf vestibulaire (3). La différence entre les signaux enregistrés avant et pendant une rotation élimine l'artéfact électrique et reflète la variation de la réponse à la SE induite par l'excitation cinétique. A ce titre, elle représente une réponse de type électrovestibulographique (EVG). Reprenant plus en détail les résultats d'une étude prémiminaire (4), nous démontrons ici que cette méthode peut être appliquée à l'enregistrement de réponses EVG sur d'autres sites : fenêtre ronde (FR), surface du cerveau, scalp. 


\section{METHODES}

L'expérimentation s'est effectuée sur des cobayes tricolores, implantés de manière chronique à l'aide d'une électrode sur la FR de l'oreille gauche et de deux vis transcranienne à la surface du cerveau. La SE étant appliquée entre la FR et l'une des deux électrodes de surface, les enregistrements sont effectués soit sur ces mêmes électrodes, soit à l'aide d'electrodes transcutanées placées sur le scalp et la mastoïde, soit encore entre la deuxième électrode de surface et la mastoïde. Le cobaye sous légère sédation (rompun) esí placé sur une table tournante, la tête orientée de façon telle que le milieu de l'axe interaural soit situé sur l'axe de rotation de la table et que le canal semi-circulaire gauche se trouve dans le plan horizontal. L'animal est soumis en permanence à des impulsions électriques alternées de durée égale à $300 \mu \mathrm{s}$, à un rythme maximum de $200 / \mathrm{s}$, induisant une excitation globale continue du système cochléo-vestibulaire. L'intensité du courant choisie, $70 \mu \mathrm{A}$, est en général suffisante pour déclencher l'ensemble des fibres cochléo-vestibulaires sans atteindre le seuil de réaction faciale.

Pour permettre une amplification suffisante du signal, et ainsi une amélioration du rapport signal/ bruit, on réalise une élimination partielle de l'artéfact de SE a l'aide d'un modèle approprié. L'artéfact étant alors réduit dans un rapport d'environ 200, l'acquisition d'un nombre total de 4000 réponses cumulées est en général suffisante pour permettre l'observation d'un potentiel EVG de qualité satisfaisante.

Dans la procédure d'enregistrement différentielle mentionnée dans l'introduction et destinée à extraire des composantes vestibulaires spécifiques de la réponse évoquée par la SE, on réalise deux enregistrements successifs, l'un dans une condition de repos, l'autre au cours d'une accélération angulaire horizontale. Les signaux moyennés entre les instants 0,75 et 3,15 s d'une phase d'accélération de $30^{\circ} \mathrm{s}^{-2}$ sont alors soustraits des enregistrements efectués au repos, faisant apparaître une réponse liée à la modulation cinétique de l'excitabilité électrique des fibres (procédure 1). Une technique alternative a consisté à utiliser un mouvement pendulaire, de période $10 \mathrm{~s}$, et à mettre en mémoire séparément les signaux cumulés pendant les phases de rotation horaires et antihoraires. Le deuxième enregistrement étant soustrait du premier, le résidu d'artéfact s'élimine, laissant voir une réponse différentielle représentant la différence d'excitabilité des fibres vestibulaires dans la condition d'accélération horaire antihoraire (procéeure 2). Pendant les deux phases d'acquisition de l'une ou l'autre procédure, un bruit blanc suffisamment intense ( $80 \mathrm{~dB}$ SPL) est appliqué en permanence à l'oreille recevant la SE, afin d'éliminer toute contribution éventuelle d'origine cochléaire. Des réponses post rotatoires ont été obtenues en effectuant un enregistrement au repos puis après un temps d'attente variable.

\section{RESULTATS}

Pour évaluer séparément les réponses EVG pouvant être associées aux deux sens de rotation, la procédure 1 a été appliquée et les réponses différentielles correspondant aux conditions "repos - accélération horaire" et "repos accélération antihoraire" ont été enregistrées sur la FR (fig. 1). Les premières comportent une onde principale positive de latence voisine de $0,4 \mathrm{~ms}$, les secondes une onde négative de latence proche de $0,3 \mathrm{~ms}$.

Comme le suggère la figure $1 \mathrm{c}$, la procédure 2 , combinant les réponses horaires et antihoraires au cours d'un même mouvement pendulaire, donne une réponse d'amplitude plus grande et dont l'artefact, soumis à une dérive de plus courte durée, est mieux éliminé. Aussi a-telle été utilisée toutes les fois que cela était possible.

Les réponses EVG obtenues sur la FR au moyen de la procédure 2 présentent une latence pratiquement invariante et une fonction de transfert sensiblement linéaire en fonction de l'accélération, un seuil de détection moyen correspondant à une vitesse de 2 a $3^{\circ} \mathrm{s}^{-1}$ et une amplitude pouvant atteindre $40 \mu \mathrm{V}$ au maximum de l'acccélération. Les réponses EVG captées dans les mêmes conditions sur le scalp (fig. 2) ou sur la surface du tronc cérébral se caractérisent par une onde négative de forme et de caractéristiques dynamiques très voisines entre elles bien que, pour les mêmes conditions
REPONSES EVG - DERIVATION FR/VERTEX C 407 SE $70 \mu A$ - SA 80 OS SPL - SV $30^{\circ} 8^{-2}$

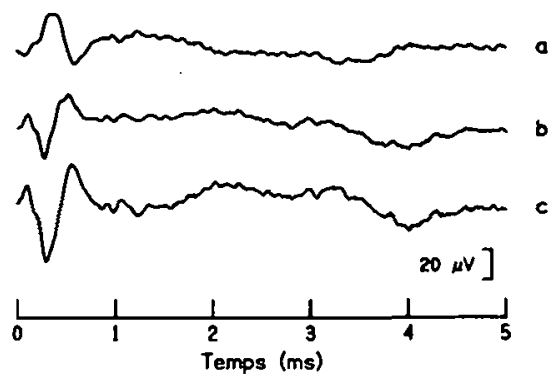

Eig.l : Procédure 1 appliquée a l'enregistrement sur la FR gauche de réponses EVG correspondant aux conditions "repos. accélération horaire" (a) et "repos - accélération antihoraire" (b). Noter les différences de polarité et de latence (respectivement 0,4 et $0,3 \mathrm{~ms}$ ) des réponses $a$ et $b$. La difference $a-b$ (courbe $c$ ), qui revient d soustraire les signaux enregistres pendant l'acceleration antihoraire de ceux enregistrés pendant l'accelération horaire. présente un pic négatif initial d'amplitude superieure a celle de $a$ ou de $b$ et de latence intermédiaire entre celles de ces deux réponses. 
d'accélération, les réponses de surface présentent un meilleur rapport signal/ bruit final. On retrouve les mêmes seuils et la même linéarité de la courbe d'amplitude que sur la FR. La courbe de latence correspond a un délai d'apparition moyen de $1,2 \mathrm{~ms}$, invariant en fonction de l'accélération. Cette réponse présente une forme plus large et le plus souvent moins diphasique que sur la FR, avec une amplitude maximale ne dépassant pas $4 \mu \mathrm{V}$. Elle est très souvent suivie d'une onde lente de latence comprise entre 3 et $4 \mathrm{~ms}$, particulièrement visible sur les enregistrements de surface.

REPONSES EVG - DERIVATION SCALP/MASTOIDE

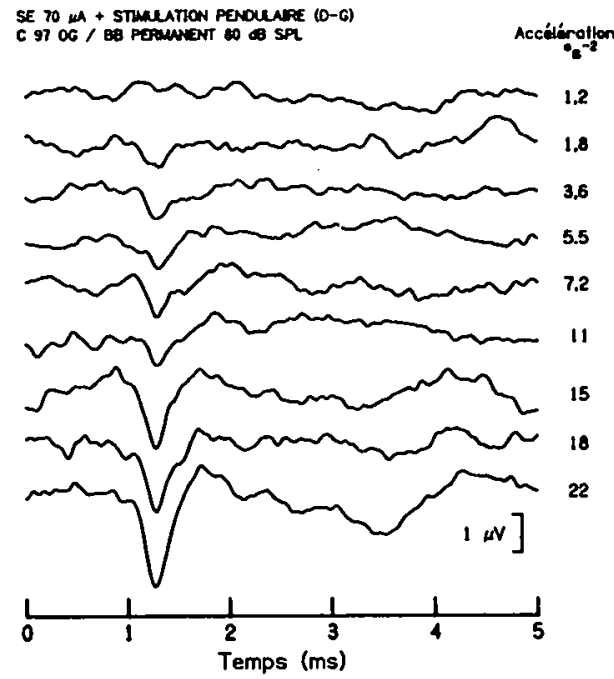

Eig. 2: Réponses EVG enregistrées sur le scalp selon la procédure 2 (cf texte) pour diverses accélerations angulaires. Les enregistrements effectués pendant les phases d'accélération antihoraire sont soustraits de ceux obtenus dans le sens horaire (total : 4000 itérations). Les nombres figurant d droite indiquent l'accéleration moyenne au cours de chaque phase d'acquisition. Période pendulaire : $10 \mathrm{~s}$.
POTENTIELS POST-ROTATOIRES G-D

C 147 SE 70 HA BQ 80 d8 SPL

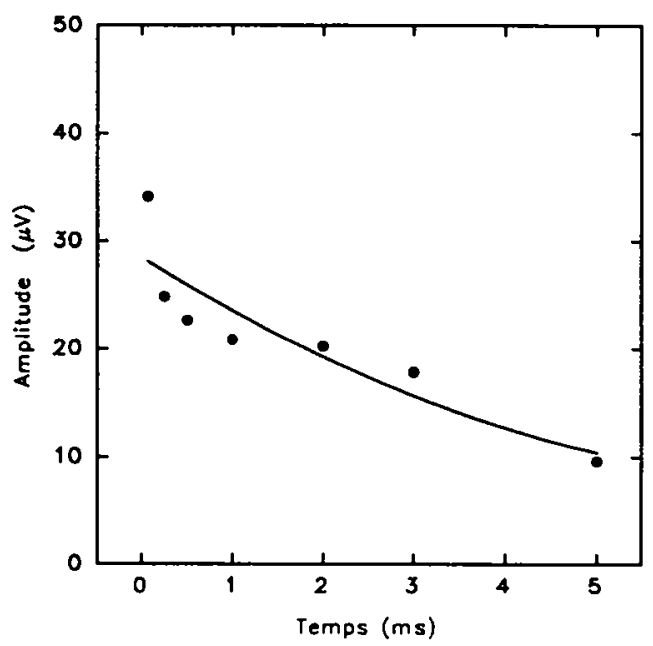

Eig. 3 : Amplitude des réponses post rotatoires sur la $F R$ d'un cobaye en fonction du temps apres l'arrêt d'une rotation de $99 \mathrm{~s}^{-1}$ maintenue pendant $10 \mathrm{~s}$ et stoppée avec une décélération de $99{ }^{\circ} \mathrm{s}^{-2}$. Les enregistrements effectues apres rotation sont soustraits de ceux effectués au repos. La procédure est répété avec un temps minimum d'attente de 8 s entre deux rotations. L'ajustement par une exponentielle décroissante indique une constante de temps de l'ordre de 4,9 s et un coefficient de determination $r^{2}=0,885$.

Des réponses post rotatoires ont été obtenues à partir d'enregistrements sur la fenêtre ronde. Les réponses combinées, résultant de la différence : enregistrement après arrêt d'une rotation antihoraire enregistrement après arrêt d'une rotation horaire, présentent une polarité négative. Les courbes temporelles, présentées ici chez un cobaye particulier (fig. 3), mettent en évidence une décroissance en fonction du temps, de constante de temps voisine de $5 \mathrm{~s}$.

Pour démontrer la spécificité vestibulaire des réponses EVG, un cobaye a été soumis à un traitement aigu consistant en une injection unique d'amikacine potentialisée par l'acide étacrynique. Les potentiels auditifs enregistrés avant, pendant et après le traitement présentent une annulation de leur amplitude en une quinzaine de minutes, suivie d'une récupération totale en 2 à 3 heures, puis d'une disparition totale irréversible en moins de 24 heures. Les potentiels EVG de surface restent inchangés au cours de l'expérimentation et les jours suivants. 


\section{DISCUSSION}

La forme des réponses dérivées par masquage vestibulaire differe significativement de celle des réponses dérivées par masquage acoustique, aussi bien sur la FR (2) que sur le tronc cérébral (5). Les formes et amplitudes sont compatibles avec les descriptions anatomiques des voies vestibulaires et auditives et suggèrent que les réponses EVG sur la FR et le tronc cérébral peuvent être associées respectivement au nerf et au noyau vestibulaires.

Une accélération angulaire dans le sens horaire exerce une action inhibitrice (6) sur l'activité des fibres du canal semicirculaire horizontal gauche, qui augmente leur excitabilité électrique et se traduit (fig. 1a) par une réponse différentielle positive, alors qu'une accélération antihoraire engendre une réponse négative (fig. 1b). La même adéquation avec les études publiées est observée en ce qui concerne la linéarité de la courbe d'amplitude (7) et la valeur du seuil de détection (8) des réponses EVG. La constante de temps cupulaire, déterminée au moyen des expériences post rotatoires, coïncide bien avec les valeurs indiquées dans la littérature (9). Le traitement par amikacine et acide étacrynique combinés montre une évolution des potentiels auditifs comparable aux observations antérieures (10, 11), correspondant à une activité cochléotoxique préservant le vestibule dont les potentiels EVG apparaissent inchangés.

Ces différentes observations confirment la spécificité vestibulaire des potentiels EVG. Le fait que la méthode puisse être appliquée à l'enregistrement de potentiels sur la FR et le scalp suggère qu'elle peut constituer une nouvelle technique d'exploration fonctionnelle du système vestibulaire, ouvrant de nouvelles perspectives d'application en particulier dans le domaine clinique.

\section{REMERCIEMENTS}

Cette étude a bénéficié d'un soutien du pôle GBM Aquitaine, contrats 88-3125 et 89-3052.

\section{REFERENCES}

(1) Ellidan J., Sohmer H., Lev S., Gay I. Short latency vestibular evoked response to acceleration stimuli recorded by skin electrodes. Ann. ORL 93 :257-261 (1984).

(2) Charlet de Sauvage R., Cazals Y., Erre J.-P., Aran J.-M. Acoustically derived auditory nerve action potentials evoked by electrical stimulation : an estimation of the waveform of single unit contribution. J. Acoust. Soc. Am. 73 : 616-627 (1983).

(3) Charlet de Sauvage R., Erre J.-P., Aran J.-M. Electrovestibulogram : first results in the guinea pig. Acta Otolaryngol. (Stockh.) $107: 489-495$ (1989).

(4) Charlet de Sauvage R. Réponses vestibulaires du tronc cérébral (VBR) enregistrées depuis le scalp sur le cobaye éveillé. In Ipsen (Ed., Paris) : XXVème Symposium français d'Oto-Neurologie, Lisbonne, 17-18 may 1991.

(5) Charlet de Sauvage R. Potentiels évoqués dans les voies auditives par stimulation électrique de l'oreille. Etude expérimentale. Rev. laryngol. (Bordeaux) 104 : 157-163 (1983).

(6) Curthoys I.S., Blanks R.H.I., Markham C.H. Semicircular canal functional anatomy in cat, guinea pig and man. Acta Otolaryngol. (Stockh.) 30 : 258-265 (1977).

(7) Goldberg, C. and J.M. Fernández. Physiology of peripheral neurons innervating semicircular canals of the squirrel monkey. I. Resting discharge and response to constant angular accelerations. J. Neurophysiol. 34 : 635-660 (1971).

(8) Melvill Jones G., Milsum J.F. Frequency analysis of central vestibular unit activity resulting from rotational stimulation of the semicircular canals. J. Physiol. 219 : 191-215 (1971).

(9) Wilson V.J., Melvill Jones G. Mammalian vestibular physiology. Ed. Wilson V.J. et Melvill Jones G. Plenum press, New York et Londres, (1979).

(10) Aran J.-M., Charlet de Sauvage R. Evolution of CM, SP and AP during etacrynic acid intoxication in the guinea pig. Acta Otolaryngol. (Stockh.) $83: 153-159$ (1977).

(11) Aran J.-M. Some functional and morphologic correlates in inner ear pathologies. Am. J. Otolaryngol. 7 : 107-116 (1986). 